\title{
Underactive Bladder; Diagnosis and Treatment at BSMMU; Single Centre Retrospective Study
}

\author{
ATM Aman Ullah ${ }^{1}$, Muhammad Hossain ${ }^{2}$, Md. Habibur Rahman ${ }^{3}$, AKM Khurshidul Alam ${ }^{4}$
}

Received: 08 - 04 - 2020

Accepted: $29-07-2020$

Conflicts of interest: None

Keywords: Underactive bladder (UAB), urodynamic examination, cystometry, lower urinary tract symptoms (LUTS), detrusor hyperreflexia with impaired contractility (DHIC), bladder outlet obstruction (BOO).

\begin{abstract}
There have been no universally agreed upon criteria and categorization of the underactive bladder (UAB). This study describes the clinical and urodynamic characteristics of $U A B$ with lower urinary tract symptoms and treatments, and attempts to provide a system of categorization.
\end{abstract}

Objective: Some patients with Lower urinary tract symptoms (LUTS) have underactive bladder and their treatment options vary subjectively. This study attempts to investigate the patient-reported experience of treatment after UDS.

Materials and methods; A retrospective study was conducted at the urology department at BSMMU from April 2017 to Dec 2019. Contraindication was Urinary Tract Infection (UTI). Patients were monitored according to follow up schedule.

Results: Total number of $U A B$ was 151. Initial complication was minimum; only two patients had UTI. Most patients improved with conservative management and CIC. The rest retain their symptoms with other treatments.

Conclusion: Conservative method and CIC is the main treatment for UAB. There are still many unknown causes for $U A B$, and many treatments are still unavailable in our country, it is still controversial whether satisfactory success is achieved in the treatment of patients with $U A B$.

\section{Introduction}

We started to work on Underactive bladder (UAB), recently which was previously considered as lower urinary tract symptoms (LUTS). During the process of UDS, we assume that it is something different in symptoms and signs as well as UDS finding. The symptom complex as urodynamically diagnosed detrusor underactivity (DU) this under-researched condition was no focus to the urologist. Presently UAB is defined as "characterised by prolonged urination time with or without the sensation of incomplete bladder emptying, usually with hesitancy, reduced sensation on filling, and a slow stream". ${ }^{1}$

Standardization reports on 2010 International Continence Society, the underactive bladder (UAB) was defined as a decrease in bladder contraction or shortening of contraction time, as a result, incomplete bladder emptying or prolonged voiding time within the normal time frame. ${ }^{2}$ In their study by Resnick et al.[3] described UAB as an emptying of the bladder in men and women with an increase in intra-abdominal pressure.

1. Associate Professor, Department of Urology, Bangabandhu Sheikh Mujib Medical University (BSMMU), Dhaka

2. Associate Professor, Department of Urology, Bangabandhu Sheikh Mujib Medical University (BSMMU), Dhaka

3. Professor, Department of Urology, Bangabandhu Sheikh Mujib Medical University (BSMMU), Dhaka

4. Professor \& Chairman, Department of Urology, Bangabandhu Sheikh Mujib Medical University (BSMMU), Dhaka

Correspondence: Dr. ATM Aman Ullah, Associate Professor, Department of Urology, Bangabandhu Sheikh Mujib Medical University (BSMMU), Dhaka, Email: amanpg@gmail.com 
According to the results of UDS in patients with nonneurogenic LUTS, UAB has been detected in $9 \%-28 \%$ of males below the age of 50 years old and $48 \%$ of those above 70 years old. the prevalence varies in older female patients, between $12 \%$ and $45 \%$, and it may be observed with impaired contractility caused by detrusor hyperreflexia. ${ }^{4,10}$

Symptoms and sign of $\mathrm{UAB}$ are nocturia/ nocturnal void, slow stream, high frequency, hesitancy, selfcatheterization, straining, urgency, urinary incontinence, immediate re-void, incomplete void, Urinary Tract Infections, post micturition dribble, intermittent void, spraying, bowel problem, acute retention, reduced sensation, and unable to pass urine.

There is no definite protocol for UAB diagnosis and treatment that will help patient's quality of life and prevent complications. Treatment targeted to empty bladder by CISC and some pharmacotherapy, very little surgical treatment.

\section{Methods}

\section{Patient selection}

We retrospectively analysed the medical records at UDS room of 395 consecutive patients who had LUTS and underwent urodynamic examinations between April 2017 and Dec 2019 to our institution. All patients underwent detailed physical examinations, medical history checks and invasive urodynamic inspections. A double-lumen transurethral catheter $(8 \mathrm{~F})$ was used for filling and to record vesical pressure. Abdominal pressure was measured with a balloon catheter (12F). Before cystometry, the remaining urine in the bladder was emptied. Saline at room temperature was infused at 10 to $40 \mathrm{~mL} / \mathrm{min}$. The type of detrusor dysfunction was evaluated during the urodynamic examination. A pressure flow study (PFS) was performed if the patient could void. Each patient underwent the urodynamic test once.

\section{Patient evaluation:}

$\mathrm{UAB}$ was diagnosed according to symptoms. The selection criteria for this study were as follows: prolonged urination time with or without a sensation of incomplete bladder discharging, hesitancy, reduced awareness on filling, and a slow stream; reduced sensation of the need to urinate, weakened or nonexistent bladder sensation, or high postvoid residual volume; and emptying bladder by catheterization or voiding with straining or increased abdominal pressure. The exclusion criteria were as follows: UTI, bladder tumour or stone before operations; urethral stricture, and pure bladder outflow obstruction (BOO).

In this study, ICS nomogram was used to grade obstruction and detrusor contractility. If there is no urine flow during the PFS, DU is defined as detrusor pressure (Pdet) d"40cm H2O.[4,5] BOO combined with DU was diagnosed by PFS and cystoscopy. We reviewed and analyzed the urodynamic findings for the included patients according to the abovementioned standards. The medical history of the selected patients was carefully reviewed to record possible etiologies that may lead to UAB.

For diagnosis, we perform a pressure-flow study (PFS). The decrease in Qmax related to BOO or poor contractility can be distinguished by PFS. BCI, as defined by Abrams, is used for the contractility of the bladder. BCI is calculated from the Qmax and Pdet@Qmax from the equation Pdet@Qmax+5Qmax. Accordingly, BCI is considered to be normal (BCI 100$150)$, strong $(\mathrm{BCI}>150)$, and weak $(\mathrm{BCI}<100) .{ }^{6}$

Female patients were required to have a detrusor pressure at maximum flow (pdetQmax) of $<20 \mathrm{~cm} \mathrm{H} 2 \mathrm{O}$ and a maximum flow rate (Qmax) of $<15 \mathrm{~mL} / \mathrm{s}$. Male patients were required to have a bladder contractility index (BCI=pdet.Qmax+5Qmax) of $<100$, and a bladder outlet obstruction index (BOOI=pdet.Qmax “ 2Qmax) of $<40.7$

The current study also corroborated recent findings by Gammie et al (8) which associated a feeling of incomplete emptying, absent, or reduced sensation and a variety of bowel issues to DU patients. A post void residual of $>30 \mathrm{ml}$ (median: $199 \mathrm{ml}$ ) was present in the majority of participants.

Idiopathic (unknown cause in young patients and normal ageing process), neurogenic (Parkinson disease, diabetes, multiple sclerosis, Guillain-Barre syndrome, spinal-lumbar disc hernia, spinal cord injury, spinal stenosis, and spinal dysraphism), myogenic [bladder outlet obstruction (BOO) and diabetes], infectious (neurosyphilis, herpes zoster, herpes simplex, and acquired immunodeficiency syndrome), and iatrogenic (pelvic surgery, radical prostatectomy, radical hysterectomy, anterior resection, and abdominoperineal resection) factors play a role in the etiology of UAB. ${ }^{9,10}$

LUTD resulting in UAB was clarified and classified by urodynamic findings. Treatment of UAB also assess by different treatment policies and follow up at one and three months PVR, voiding history, and infections. 


\section{Treatments:}

Here is no definite protocol for UAB treatments for improve quality and prevention of complications. Treatments options are as follows. Table I.

\section{Table-I}

Treatment of underactive bladder and promising alternatives

Conservative methods and clean intermittent catheterization

Pharmacotherapy

Alpha-blockers

Cholinesterase inhibitors (distigmine, pyridostigmine, neostigmine)

Muscarinic agonists (bethanechol and carbachol)

Prostaglandin E2

Acotiamide

Surgical methods

Sacral nerve stimulation-electrical stimulation

Other surgical methods

Injections into the external sphincter

Surgeries for bladder outlet obstruction

Reduction cystoplasty

Latissimus dorsi detrusor myoplasty

Stem cell and gene therapies

\section{Statistical analysis}

Data are expressed as a percentage (\%).

\section{Results}

During the period from April 2017 to December 2019, there were 395 consecutive patients with LUTS who sought evaluation at the urodynamic center in our department. A total of 151 patients with UAB (91 men and 60 women; $10-83$ years old) were included into the study cohort. Many risk factors that cause UAB, were found by analyzing the related etiology (Table $2)$. In some cases, the factors causing $U A B$ remain unidentified, therefore due to a lack of evident etiology, $\mathrm{UAB}$ can be classified as idiopathic. It accounted for $20 \%$ (30) of the cases. Patients with BOO leading to UAB had myogenic UAB, which accounted for $4.6 \%$ (7) of cases. Patients in whom risk factors leading to $\mathrm{UAB}$ acted on the neurologic etiology had neurogenic UAB, which accounted for $62.9 \%$ (95) of cases, infections $2.6 \%$ (4), and iatrogenic $9.9 \%$ (15). The nervous system factors contributed to the major of UAB cases. Some UAB had more than two etiological factors. $\mathrm{UAB}$ without coexisting urological conditions $(\mathrm{n}=19)$, $\mathrm{UAB}+$ coexisting urological conditions $(n=25)$ over active bladder $(\mathrm{OAB})$, bladder outlet obstruction (BOO), Stress, and urgency incontinence.

The urodynamic findings present absent detrusor contraction or reduced detrusor contractility (with or without detrusor overactivity [DO] throughout the filling phase). The patients might have urine flow or complete urinary retention. Among $151 \mathrm{UAB}$ patients, the percentage of detrusor hyperreflexia with impaired contractility (DHIC), DU, and acontractile detrusor (AcD) was $3.3 \%,(5), 58 \%,(88)$ and $38.4 \%$ (58), respectively. The symptoms were different with the 3 groups, as follows: DU had typical symptoms mentioned above; DHIC with UAB had OAB symptoms; and $\mathrm{AcD}$ had urinary retention and history of catheterization or voiding with increased abdominal pressure or with straining.

Conservative Methods and CIC: Patients with symptoms, such as storage and voiding symptoms and increased amount of PVR, are detected. All patients after UDS, such as voiding symptoms and high amount of PVR, are detected, they were included in the treatment plan. These group of patients counselled, water restriction to 2-2.5 litre/ day according to

Table-II

Etiological classification of underactive bladder in 151 patients.

\begin{tabular}{lll}
\hline Type & Possible causes & $\mathrm{N}(\%)$ \\
\hline Idiopathic & Aging, Young patient unknown causes & $30(20 \%)$ \\
Myogenic & DM TURBT BOO & $7(4.6 \%)$ \\
Neurogenic & CVD, Spinal Injury, Spinal TB, Parkinsonism, DM & $95(62.9 \%)$ \\
Iatrogenic & Abdominal hysterectomy, Pelvic surgery, Abdominoperineal resection & $15(9.9 \%)$ \\
Infectious & Herpes simplex and Herpes zoster & $4(2.6 \%)$ \\
\hline
\end{tabular}


occupation, avoid constipation, dietary advice, timed, double, or triple voiding. The amount of PVR can be decreased using the Valsalva or Crede maneuver. However, these are contraindicated in patients for vesicoureteral reflux, high intravesical pressure, and vagal reflex. Patients were advice for medical treatment alpha blocker and muscarinic drugs. ${ }^{11}$

A few patients have difficulty in continuing catheterization, and they were assisted by family members. Generally, Nelaton catheter 12-16 French was used, and the daily number of CIC was between 4 and 6 times. Ideally, the amount of urine should not exceed 400-500 cc at every application of CIC.

None of pharmacotherapy, oral medications used in the UAB is completely effective. The use of drug treatment is to increase intravesical pressure, detrusor contractility as well as decreasing bladder outlet resistance. These agents are used as alpha-blockers, they reduce urethral outlet pressure, and muscarinic ago-nists (bethanechol and carbachol). ${ }^{12,13}$ Alphablockers used for patients with chronic BOO have a risk of development of UAB. This is particularly evident for older patients with benign enlarged prostates (BPH). Alpha-blockers decrease BOO-related symptoms and facilitate emptying in patients with neurogenic lower urinary tract dysfunction and $\mathrm{BPH} .{ }^{12}$ At the same time, it is female patients with voiding difficulty.

Muscarinic agonists (bethanechol and carbachol), specially Bethanechol, acts similar to acetylcholine and stimulates muscarinic receptors, as a result increasing detrusor tone, and contractility of the bladder. It should be taken at an empty stomach three or four times per day. We did Surgeries for bladder outlet obstruction after cystoscopy like BNI, BNR, and TURP. Two patients developed UTI and successfully managed by sensitive antibiotics.

We had no experience with these following treatments with Prostaglandin E2, Acotiamide. Surgical methods: sacral nerve stimulation-electrical stimulation, onabotu-linumtoxinA injections into the external sphincter, reduction cystoplasty, latissimus dorsi detrusor myoplasty, and stem cell and gene therapies.

\section{Discussion}

Some studies have focused on the etiologic categorization of UAB. Osman et al proposed 4 categorical types based on etiologic factors which are idiopathic, neurogenic, myogenic, and iatrogenic. ${ }^{14}$
However iatrogenic factors should be categorized as neurogenic as it often results in injury to the peripheral pelvic plexus. We proposed a similar but distinct classification based on the possible causes (idiopathic, myogenic, neurogenic, and integrative). While van Koeveringe et $\mathrm{al}^{15}$ described idiopathic UAB as an agelinked decline in detrusor contractility or other imprecise causes, but $\mathrm{BOO}$ along with age was included. $\mathrm{Kuo}^{16}$ defined idiopathic $\mathrm{UAB}$ as no evident neuropathy, no functional or anatomic bladder outlet obstruction, low or no Pdet combined with a maximum flow rate, and a large postvoid residual volume or urinary retention. We consider that individuals with whom the central cause was an age-linked decline in detrusor contractility (excluding BOO) or other unknown causes and with symptoms of UAB might be labeled as idiopathic. Cucchi et $\mathrm{al}^{17}$ thought that $\mathrm{UAB}$ was most often idiopathic, although it might be caused by other causes. However, we found that only $20 \%(30 / 151)$ of patients were idiopathic in the current study. Long-term BOO could not only cause the structural changes of detrusor muscle cells, but also a reduction in detrusor blood flow. ${ }^{18}$ Chronic overexpansion causes injury to postsynaptic parasympathetic ganglia in bladder wall, and stimulates muscle fiber damage, which leads to decompensation of detrusor function and advances to DU. Lee et al[19] reported that $37 \%$ to $47 \%$ of patients with benign prostate hyperplasia had impaired detrusor contractility. In the current study, we classified $\mathrm{UAB}$ subjective to $\mathrm{BOO}$ as myogenic. Myogenic UAB may result from altered excitation-contraction coupling mechanisms of detrusor muscle cells and result in reduced self-directed activity of the bladder. ${ }^{15}$ Neurogenic UAB may result from direct changes in the efferent limb of the micturition reflex, the afferent signals initiating the reflex, and the integrative control. ${ }^{15}$ In the current study, we reviewed the potential factors leading to neurogenic UAB (Table II), including nervous system factors, iatrogenic factors and infectious factors involving the nervous system. Neurogenic UAB represented the largest proportion of this study cohort $62.9 \%$ (95/151).

SCI and PLID was an important factor which accounted for $29.1 \%$ (44/151) of the Neurogenic UAB. Most patients showed AcD and had urinary retention. The emptying of the bladder was executed by catheterization to avoid damage to the upper urinary tract. Although other neurologic problems, such as Parkinson disease, AIDS, neurosyphilis, and GuillainBarre syndrome, were not listed by the study, other 
neurologic problems could also be contributing factors to UAB.(20)Although there was a relatively small percentage $(1.3 \%[22 / 1726])$, we found that some patients who had a diagnosis of UAB could have 2 or more etiologies. Given that the etiologies resulting to $\mathrm{UAB}$ are not well-defined at present, we suggest a categorization of 3 types based on urodynamic findings and symptoms (DU, AcD, and DHIC). DU means that detrusor contraction function is impaired. Patients with DU have or no urine flow (retention) or are able to void (emptying completely or incompletely) throughout the urodynamic inspection. AcD indicates that there are no detrusor contractions during urodynamic studies. The logical conjecture is that AcD is the extremity of DU during its progression. [10] Where patients with $\mathrm{AcD}$ have urinary retention and the emptying of the bladder is achieved by increased abdominal pressure, or catheterization. Most patients $(58 \%,(88))$ in this study had characteristics of DU. DHIC is defined as DO during the filling phase and underactive detrusor contractions during the voiding phase, thus a weak detrusor. ${ }^{21}$ DHIC presents as a combination of $\mathrm{DU}$ and $\mathrm{DO}(\mathrm{UAB}$ and $\mathrm{OAB}$ symptoms). We found that $3.3 \%,{ }^{5}$ of patients with UAB demonstrated urodynamic manifestations of DHIC. It is important to identify DHIC with urodynamic testing because treating patients with anticholinergic drugs may worsen bladder emptying leading to urine retention and/or urinary tract infection. ${ }^{22}$

For patients with significant PVR and urinary retention; CIC would be recommended. Informing the patient and his/her family about the advantages of this practice is important for patient compliance. ${ }^{25}$ The European Association of Urology (EAU) guidelines indicate that CIC is the standard of care in the management of the patient group who cannot effectively empty their bladders. For this group of patients, due to complications; it is emphasized that suprapubic cystoscopy and transurethral catheterization be evaded and may result to an increased risk of encrustation, leakage, discomfort, UTI, urethral erosion, and bladder spasm..$^{23}$ It should not be disre-garded that both CIC and indwelling catheters will reduce the QoL of patients and cause emotional stress.

Comparably, the efficiency of alpha-blockers has been confirmed in female patients with subnormal maximal voiding velocities and voiding difficulty. In a trial by Chang et al. ${ }^{24}$ after treatment with $0.2 \mathrm{mg}$ tamsulosin for 6 weeks, $35.1 \%$ of the patients had decreased voiding symptom scores by $>50 \%$, and Qmax increased by $>30 \%$.
Bethanechol, this agent has side effects, such as nausea, vomiting, diarrhea, gastrointestinal system cramps, sweating, bronchospasm, and visual disturbances. ${ }^{25}$

Sacral nerve stimulation-electrical stimulation: Studies have shown that sacral nerve stimulation (SNS) and intravesical electrical stimulation are useful in selected patients. Urinary voiding functions and retention occur with different mechanisms and times. Structures that stimulate both mechanisms are placed at S2-S4 levels. ${ }^{26}$

Injections into the external sphincter: onabotulinumtoxinA injections into the detrusor muscle have been approved by the FDA, there is insufficient evidence data to support onabotulin-umtoxin A injections. Despite many studies, there is no standard dosage schedule. ${ }^{27}$

The regeneration potentials of the urethral smooth muscle cells and bladder are constrained. For bladder repair research is particularly focused on multipotent stem cells. The stem cells used are generally obtained from skeletal muscles. The use of the skeletal muscle has satellite cells that help tissue repair in case of cell damage in the skeletal muscle cells as opposed to the smooth muscle cells. ${ }^{28}$

Gene therapy is expected to be more involved in the treatment options in the future.

This study had some limitations. This study was a single center, retrospective data analysis. Various factors may have impacted the outcome, such as novel environment intraurethral catheter and patient position, which may stimulate the urodynamic results, instigating more DU. A multicenter, prospective study should be performed to validate this classification of $\mathrm{UAB}$.

\section{Conclusion:}

Conservative method and CIC is main treatment for UAB. There are still many unknown causes for UAB, and many treatments are still unavailable at our country, it is still controversial whether satisfactory success is achieved in the treatment of patients with $\mathrm{UAB}$.

Due to its intricacy of clinical categorization UAB has raised substantial controversy in many areas at present. Based on the large sample data, we classified UAB into 5 types based on etiology, and into 3 types based on symptoms and urodynamic findings in this retrospective study. The classification can provide a reasonable basis for future research. 


\section{References:}

1. Chapple CR, Osman NI, Birder L, et al. The underactive bladder: a new clinical concept? Eur Urol 2015;68:351-3.

2. Haylen BT, de Ridder D, Freeman RM, Swift SE, Berghmans B, Lee J, et al. An International Urogynecological Association (IUGA)/ International Continence Society (ICS) joint report on the terminology for female pel-vic floor dysfunction. Neurourol Urodyn 2010;29:4-20.

3. Resnick NM, Yalla SV, Laurino E. The pathophysiology of urinary incontinence among institutionalized elderly persons. N Engl J Med 1989;320:1-7.

4. Jeong SJ, Kim HJ, Lee YJ, et al. Prevalence and clinical features of detrusor underactivity among elderly with lower urinary tract symptoms: a comparison between men and women. Korean J Urol 012;53:342-8.

5. Thomas AW, Cannon A, Bartlett E, et al. The natural history of lower urinary tract dysfunction in men: the influence of detrusor underactivity on the outcome after transurethral resection of the prostate with a minimum 10-year urodynamic follow-up. BJU Int 2004;93:745-50.

6. Abrams P. Bladder outlet obstruction index, bladder contractility in-dex and bladder voiding efficiency: Three simple indices to define bladder voiding function. BJU Int 1999;84:14-5.

7. Uren AD, Cotterill N, Harding C, et al. Qualitative exploration of the patient experience of underactive bladder. Eur Urol.2017;72(3):402 407.

8. Gammie A, Kaper M, Dorrepaal C, Kos T, Abrams P. Signs and symptoms of detrusor underactivity: an analysis of clinical presentation and urodynamic tests from a large group of patients under-going pressure flow studies. Eur Urol 2016;69:361-9.

9. Haylen BT, de Ridder D, Freeman RM, Swift SE, Berghmans B, Lee J, et al. An International Urogynecological Association (IUGA)/ International Continence Society (ICS) joint report on the terminology for female pel-vic floor dysfunction. Neurourol Urodyn 2010;29:4-20.

10. Osman NI, Chapple CR, Abrams P, Dmochowski R, Haab F, Nitti V, et al. Detrusor underactivity and the underactive bladder: A new clini-cal entity? A review of current terminology, definitions, epidemiol-ogy, aetiology, and diagnosis. Eur Urol 2014;65:389-98.

11. Miyazato M, Yoshimura N, Chancellor MB. The other bladder syn-drome: Underactive bladder. Rev Urol 2013;15:11-22.

12. Kim DK. Current pharmacological and surgical treatment of under-active bladder. Investig Clin Urol 2017;58:S90-8.

13. Chancellor MB, Kaufman J. Case for pharmacotherapy develop-ment for underactive bladder. Urology 2008;72:966-7.

14. Osman NI, Esperto F, Chapple CR. Detrusor underactivity and the underactive bladder: A systematic review of preclinical and clinical studies. Eur Urol 2018;74:633-43.

15. van Koeveringe GA, Vahabi B, Andersson KE, et al. Detrusor underactivity: a plea for new approaches to a common bladder dysfunction. Neurourol Urodyn 2011;30:723-8.

16. Kuo HC. Recovery of detrusor function after urethral botulinum A toxin injection in patients with idiopathic low detrusor contractility and voiding dysfunction. Urology 2007;69:57-61.

17. Cucchi A, Quaglini S, Rovereto B. Development of idiopathic detrusor underactivity in women: from isolated decrease in contraction velocity to obvious impairment of voiding function. Urology 2008;71:844-8.

18. Belenky A, Abarbanel Y, Cohen M, et al. Detrusor resistive index evaluated by Doppler ultrasonography as a potential indicator of bladder outlet obstruction. Urology 2003;62:64750 .

19. Lee JG, Shim KS, Koh SK. Incidence of detrusor underactivity in men with prostatism older than 50 years. Korean J Urol 1999;40:347-52.

20. Miyazato M, Yoshimura N, Chancellor MB. The other bladder syndrome: underactive bladder. Rev Urol 2013;15:11-22.

21. Stav K, Shilo Y, Zisman A, et al. Comparison of lower urinary tract symptoms between women with detrusor overactivity and impaired contractility, and detrusor overactivity and preserved contractility. J Urol2013;189:2175-8. 
22. Kitta T, Mitsui T, Kanno Y, et al. Postoperative detrusor contractility temporarily decreases in patients undergoing pelvic organ prolapse surgery. Int J Urol 2015;22:201-5.

23. Blok B, Pannek J, Castro-Diaz D, Del Popolo G, Groen J, Hamid R, et al. EAU Guidelines on Neuro-Urology. (C) European Association of Urology (EAU), 2018.

24. Chang SJ, Chiang IN, Yu HJ. The effectiveness of tamsulosin in treating women with voiding difficulty. Int J Urol 2008;15:981-5

25. Miyazato M, Yoshimura N, Chancellor MB. The other bladder syn-drome: Underactive bladder. Rev Urol 2013;15:11-22.
26. Chancellor MB, Chartier-Kastler EJ. Principles of sacral nerve stim-ulation (SNS) for the treatment of bladder and urethral sphincter dysfunctions. Neuromodulation 2000;3:16-26

27. Chang YH, Siu JJ, Hsiao PJ, Chang CH, Chou EC. Review of under-active bladder. J Formos Med Assoc 2018;117:178-84.

28. Lee JY, Qu-Petersen Z, Cao B, Kimura S, Jankowski R, Cummins J, et al. Clonal isolation of muscle-derived cells capable of enhancing muscle re-generation and bone healing. J Cell Biol 2000;150:1085-100. 